This article was originally submitted to the February 2013 issue on low-grade gliomas;

however, a publication moratorium on data from The Cancer Genome Atlas delayed publication until this time.

\title{
The Cancer Genome Atlas expression profiles of low-grade gliomas
}

\author{
David D. Gonda, M.D., ${ }^{1}$ Vincent J. Cheung, M.D., ${ }^{1}$ Karra A. Muller, M.D., Ph.D., ${ }^{2}$ \\ Amit Goyal, B.S., ${ }^{1}$ Bob S. Carter, M.D., Ph.D., ${ }^{1}$ and Clark C. Chen, M.D., Ph.D. ${ }^{1}$ \\ Departments of ${ }^{1}$ Neurosurgery and ${ }^{2}$ Neuropathology, University of California, San Diego, California
}

\begin{abstract}
Differentiating between low-grade gliomas (LGGs) of astrocytic and oligodendroglial origin remains a major challenge in neurooncology. Here the authors analyzed The Cancer Genome Atlas (TCGA) profiles of LGGs with the goal of identifying distinct molecular characteristics that would afford accurate and reliable discrimination of astrocytic and oligodendroglial tumors. They found that 1) oligodendrogliomas are more likely to exhibit the glioma$\mathrm{CpG}$ island methylator phenotype (G-CIMP), relative to low-grade astrocytomas; 2) relative to oligodendrogliomas, low-grade astrocytomas exhibit a higher expression of genes related to mitosis, replication, and inflammation; and 3) low-grade astrocytic tumors harbor microRNA profiles similar to those previously described for glioblastoma tumors. Orthogonal intersection of these molecular characteristics with existing molecular markers, such as IDH1 mutation, TP53 mutation, and 1p19q status, should facilitate accurate and reliable pathological diagnosis of LGGs. (http://thejns.org/doi/abs/10.3171/2012.12.FOCUS12351)
\end{abstract} \author{
KEY WoRDS $\bullet$ astrocytoma $\bullet$ oligodendroglioma $\bullet$ oligoastrocytoma
low-grade glioma
}

$\mathrm{D}$ IFFERENTIATING between LGGs of astrocytic and oligodendroglial origin remains a major challenge in neurooncology. ${ }^{8,23,28}$ Overcoming this challenge is paramount. Astrocytic and oligodendroglial tumors exhibit distinct natural histories, biologies, and therapeutic responses. ${ }^{14,33}$ As such, our ability to appropriately care for patients afflicted with LGGs and advance the standard of care greatly depends on accurate pathological diagnosis.

It is increasingly apparent that histological features are insufficient for consistently discriminating between the astrocytic and oligocytic LGG (Fig. 1). ${ }^{8,25}$ There is a general consensus in terms of the need for molecular tools in this regard. ${ }^{24,30}$ While great strides have been made toward the development of these tools with the discovery of TP53 mutations and 1p19q deletions, reliable molecular diagnosis remains elusive for the $30 \%-50 \%$ of LGGs that do not harbor these molecular markers. ${ }^{11,18}$

We propose that the discrimination of astrocytic and oligocytic LGGs can be facilitated by comprehensive molecular analysis of these tumors. To this end, we analyzed the publicly available multiplatform molecular analysis of LGGs from TCGA. ${ }^{1}$

\section{Methods \\ Determination of G-CIMP Status}

Methylation data related to glioblastoma and LGG

Abbreviations used in this paper: $\mathrm{G}-\mathrm{CIMP}=$ glioma-CpG island methylator phenotype; GBM = glioblastoma multiform; $\mathrm{LGG}=$ low-grade glioma; miRNA = microRNA; TCGA = The Cancer Genome Atlas. were downloaded from TCGA data portal. The Level II data have been preprocessed and normalized per probe set by TCGA working group. Using the methods described by Noushmehr et al., ${ }^{20}$ we identified the top 1427 probes whose DNA methylation beta values varied most across the glioblastoma and LGG samples. The glioblastoma and LGG data sets were grouped and classified by k-medians clustering (number of clusters 3 ) of the selected probe sets. All clustering of the data and construction of the heatmaps were performed using the TM4 MultiExperiment Viewer (version 4.7.4, Dana-Farber Cancer Institute). ${ }^{27}$

\section{Determination of TCGA Subtype}

Level III mRNA expression data were obtained from TCGA data portal for 27 LGG and 603 GBM specimens. Level III data consisted of expression calls for genes per sample following processing and normalization including $\log 2$ transformation of the gene expression levels. Data from the LGG and GBM samples were compiled into a single data set, and relative gene expression values were calculated by subtracting from the gene estimate the mean expression values across patients and then dividing the difference by the standard deviation across all patients. Using the 840 gene signature reported by Verhaak et al., ${ }^{32}$ we classified the tumors into proneural, neural, classical, and mesenchymal subtypes by k-medians clustering (number of clusters 4).

\section{Differential Gene Expression Analysis}

Level III mRNA expression data from Agilent Technologies G4502A-07 microarrays were obtained from 


\section{D. Gonda et al.}
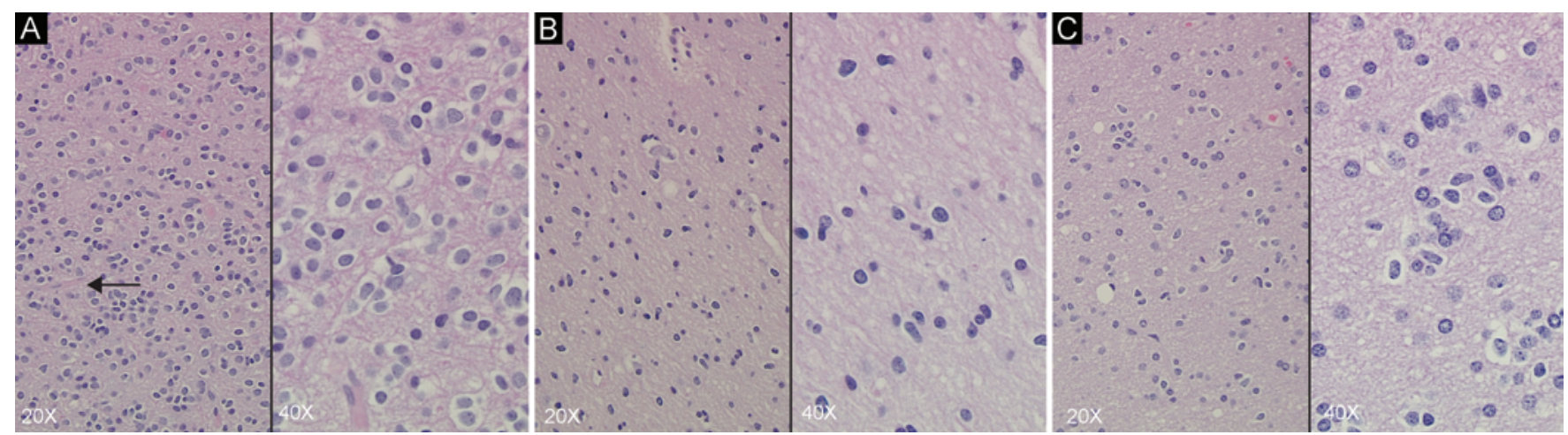

FIG. 1. Photomicrographs demonstrating common histopathologies of LGGs. A: Classic oligodendrogliomas, WHO Grade II, display uniform cellularity and uniformly round nuclei with small but distinct nucleoli that sit within perinuclear halos (the socalled fried-egg appearance). The infiltrative nature is suggested by myelinated axons coursing in the background of tumor cells. Thin "chicken-wire" vasculature is often seen (arrow). B: Low-grade diffuse (infiltrating) astrocytomas, WHO Grade II, are most commonly characterized by a fibrillary pattern, as shown here. Fibrillary astrocytomas have more than twice-normal cellularity attributable to pleomorphic astrocytes varying in size and shape. These cells often show nuclear elongation, angulation, hyperchromasia, pleomorphism, and enlargement. Again, the myelinated axons throughout the background confirm the tumor is infiltrating. C: Low-grade oligoastrocytomas, WHO Grade II, are controversial entities within neuropathology. Multiple patterns have been suggested to describe mixed oligoastrocytomas, and one suggested pattern is represented in this figure, with individual cells having both astrocytic and oligodendroglial features. Cells within this tumor have the nuclear elongation, angulation, and enlargement of an astrocytoma but are sitting within perinuclear halos like an oligodendroglioma. $\mathrm{H} \& \mathrm{E}$.

TCGA data portal. The mean fold change analysis between the low-grade astrocytomas and oligodendrogliomas was performed on all genes in the full 17813 gene data set. Genes that demonstrated mean differential expression of at least 2-fold were kept as interesting genes for further analysis. Gene lists of interest were uploaded and analyzed using the online gene ontology tool DAVID 6.7 (National Institute of Allergy and Infectious Diseases, $\mathrm{NIH}$, http://david.abcc.ncifcrf.gov/)., ${ }^{9,10}$

Level III miRNA expression data and their corresponding clinical data from 47 LGG samples were downloaded from TCGA data portal. The miRNA quantification data from each sample were combined into a single data set, and then mean fold change values for each gene were calculated by comparing astrocytomas with oligodendrogliomas. The miRNAs that demonstrated a mean fold change of at least 2 were kept as potentially interesting miRNAs for further analysis. The miRNAs were organized according to unsupervised hierarchical clustering, and the samples were grouped according to histological diagnosis.

\section{Results}

\section{The G-CIMP Status}

The G-CIMP status was determined for 24 LGGs (composing 11 oligodendrogliomas, 8 oligoastrocytomas, and 5 astrocytomas), $294 \mathrm{GBMs}$, and 2 matched normal specimens. Seventeen (70.8\%) of the 24 LGGs were GCIMP-positive tumors, compared with only $8.5 \% \mathrm{G}-$ CIMP positivity among the 294 GBMs analyzed (Fig. 2 left). All oligodendrogliomas (11 of 11) exhibited GCIMP positivity (Fig. 2 right). Only 1 of 5 astrocytomas was G-CIMP positive, whereas the distribution among oligoastrocytomas was more evenly divided, with 6 of 9 being G-CIMP positive. Both matched normal specimens were negative for the hypermethylation phenotype.

\section{Analysis of $m R N A$}

Twenty-seven LGG specimens were classified according to TCGA subtype by the expression pattern of the 840 gene signature defined by Verhaak et al..$^{32}$ The LGGs analyzed included 8 oligodendrogliomas (4 WHO Grade II, 4 WHO Grade III), 9 oligoastrocytomas (2 WHO Grade II, 7 WHO Grade III), and 10 astrocytomas (1 WHO Grade II, 9 WHO Grade III). All but one of the characterized LGGs had gene expression characteristics of the proneural glioma subtype (Fig. 3 left). The lone exception was a WHO Grade II oligodendroglioma in a patient whose tumor's molecular characteristics were consistent with the mesenchymal subtype. Notably, this lone patient with the mesenchymal oligodendroglioma died within 2 years of the surgical diagnosis, demonstrating a more aggressive course than is typically seen in oligodendroglioma tumors.

Differential gene expression analysis was then performed comparing oligodendrogliomas with astrocytomas (Fig. 3 right). The oligoastrocytomas were not included in this analysis since they represent a less descript subtype with overlapping histological components of the other 2 tumor types. Using a 2-fold mean differential expression cutoff, 338 genes were found to be upregulated and 283 genes were downregulated in the astrocytomas relative to the oligodendrogliomas. Gene ontology analysis of the 338 upregulated genes yielded gene ontology terms related primarily to mitotic functions and the inflammatory response, for example, mitosis, cell cycle phase, immune response, and inflammatory response (Table 1). The most significant cluster had an enrichment score of 8.64 and contained 54 of the 338 upregulated genes, many of which were mRNAs coding for inflammatory cell receptors, ligands, and major histocompatibility complexes. The cluster enrichment score for mitotic genes was 6.37, and this cluster consisted of 61 of the 338 upregulated genes. 


\section{The Cancer Genome Atlas expression profiles of low-grade gliomas}
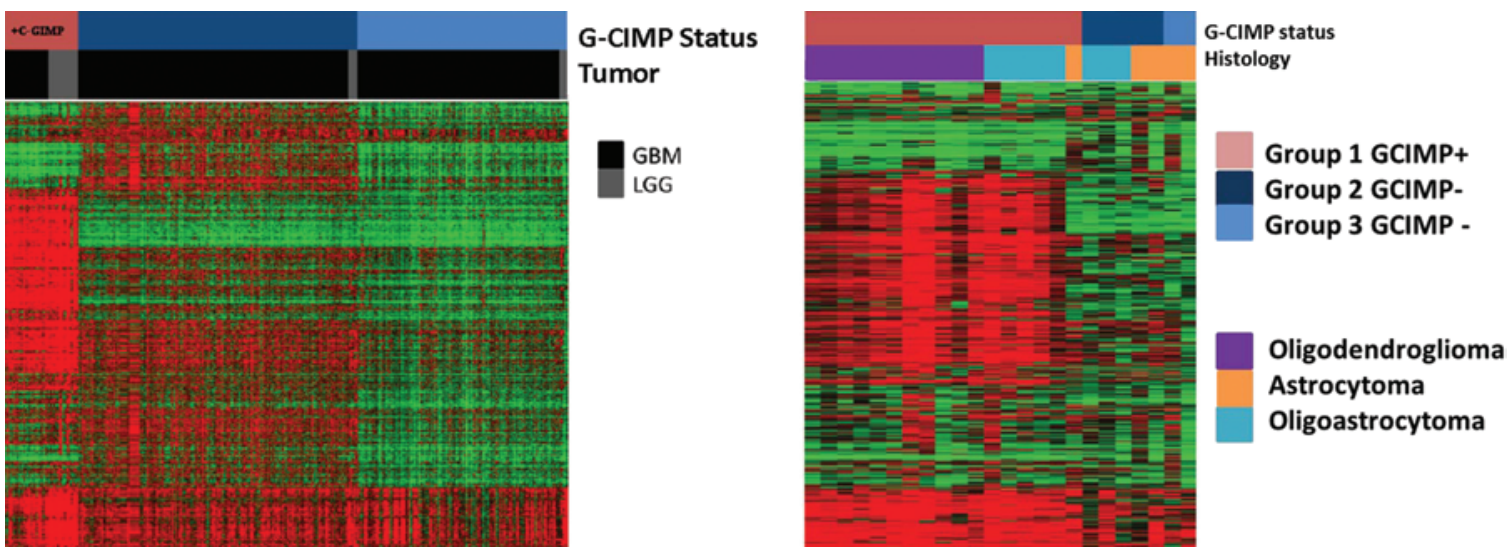

FIG. 2. Clustering of TCGA LGG and GBM tumors identifies G-CIMPs. After removing probe sets targeting the sex chromosomes, the 1427 Infinium DNA methylation probes, whose DNA methylation beta values varied the most across 294 TCGA GBM samples, were analyzed. The DNA methylation clusters are distinguished with a color code at the top panel: pink, G-CIMPpositive Cluster 1; dark blue, G-CIMP-negative Cluster 2; and light blue, G-CIMP-negative Cluster 3. Left: Heatmap of the DNA methylation data from 24 LGG samples combined with 294 GBM samples revealing the G-CIMP status after k-medians clustering (number of clusters 3 ) of the sample order. The level of DNA methylation (beta value) for each probe in each sample is represented by a color scale, with red indicating higher levels of methylation and green showing lower levels. Each sample is color labeled as described in the key for its tumor histology type (LGG vs GBM). Right: Heatmap of the 24 LGG samples clustered alone by k-medians clustering (number of clusters 3) revealing the G-CIMP status. Again, each sample is color labeled as described in the key for its tumor histology type (oligodendroglioma vs astrocytoma vs oligoastrocytoma).

\section{Analysis of miRNA}

We also compared miRNA expression differences between 23 oligodendrogliomas (12 WHO Grade II, 11 WHO Grade III) and 24 astrocytomas (2 WHO Grade II, 22 WHO Grade III) as obtained from TCGA microarray data (Fig. 4). Nine miRNAs were upregulated and
28 were downregulated more than 2-fold within the astrocytomas relative to the oligodendrogliomas (Table 2). The upregulated list included miRNAs known to be upregulated in GBMs as well as a variety of nonneural tumors such as miR-21. ${ }^{5}$ Downregulated miRNAs included those associated with neuronal differentiation, which are
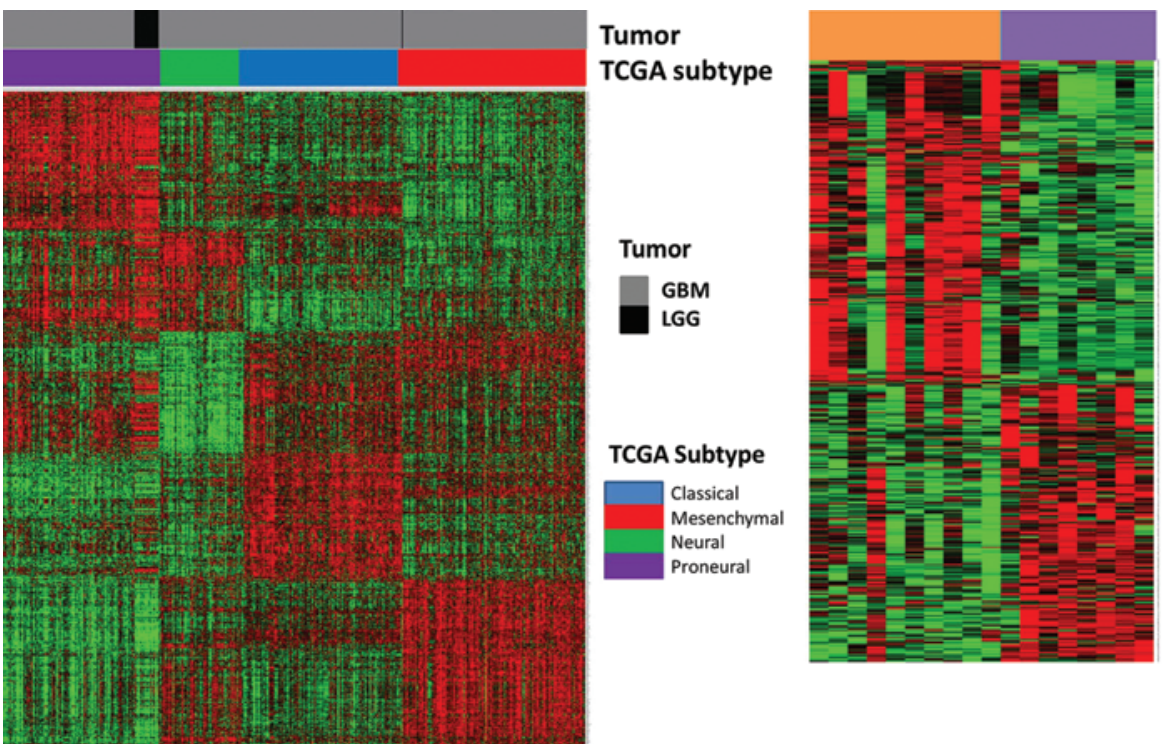

Histology

Oligodendroglioma

Astrocytoma

FIG. 3. Left: Gene expression data identify the 4 TCGA subtypes. Using the 840 gene list defined by Verhaak et al., we ordered samples based on subtype predictions after k-medians clustering (number of clusters 4). Tumor types (GBM vs LGG) are distinguished with a color code at the top panel: gray, GBM; and black, LGG. The Cancer Genome Atlas subtypes are defined in the center panel: red, mesenchymal; blue, classical; green, neural; and purple, proneural. Heatmap showing the level of mRNA gene expression normalized for each gene using a color scale, with red representing higher levels of expression and green indicating lower levels. Right: Differential gene expression of astrocytomas and oligodendrogliomas. Direct comparison between the 2 tumor types revealing 621 mRNAs with a minimum mean fold change of 2 . Tumor types (oligodendroglioma vs astrocytoma) are distinguished with a color code at the top panel: orange, astrocytoma; and purple, oligodendroglioma. Heatmap showing the level of mRNA gene expression normalized for each gene using a color scale, with red representing higher levels of expression and green indicating lower levels. 
D. D. Gonda et al.

TABLE 1: Resulting gene ontology terms using the online tool DAVID for functional analysis of the 338 genes upregulated more than 2-fold in astrocytomas as compared with oligodendrogliomas

\begin{tabular}{|c|c|c|c|}
\hline Gene Ontology Terms & Gene Count & p Value* & Corrected $p$ Value $†$ \\
\hline \multicolumn{4}{|l|}{ Functional Cluster 1, Enrichment Score 8.64} \\
\hline immune response & 41 & $9.00 \mathrm{E}-12$ & 8.40E-09 \\
\hline defense response & 37 & $8.70 \mathrm{E}-11$ & $2.30 \mathrm{E}-08$ \\
\hline inflammatory response & 25 & 1.80E-09 & $3.30 \mathrm{E}-07$ \\
\hline response to wounding & 29 & $1.20 \mathrm{E}-07$ & $1.90 \mathrm{E}-05$ \\
\hline inflammatory response & 11 & $3.80 \mathrm{E}-07$ & $3.10 \mathrm{E}-05$ \\
\hline \multicolumn{4}{|l|}{ Functional Cluster 2, Enrichment Score 6.37} \\
\hline M phase & 29 & $2.40 \mathrm{E}-12$ & 4.50E-09 \\
\hline mitosis & 21 & 1.40E-11 & $4.50 \mathrm{E}-09$ \\
\hline cell cycle phase & 31 & $2.20 \mathrm{E}-11$ & 1.40E-08 \\
\hline mitosis & 23 & $2.70 \mathrm{E}-11$ & $1.20 \mathrm{E}-08$ \\
\hline nuclear division & 23 & $2.70 \mathrm{E}-11$ & $1.20 \mathrm{E}-08$ \\
\hline M phase of mitotic cell cycle & 23 & $3.90 \mathrm{E}-11$ & $1.40 \mathrm{E}-08$ \\
\hline organelle fission & 23 & $6.00 \mathrm{E}-11$ & $1.80 \mathrm{E}-08$ \\
\hline cell cycle process & 34 & $6.20 \mathrm{E}-10$ & 1.40E-07 \\
\hline mitotic cell cycle & 27 & $1.00 \mathrm{E}-09$ & 2.10E-07 \\
\hline cell division & 21 & $9.40 \mathrm{E}-09$ & $1.50 \mathrm{E}-06$ \\
\hline cell cycle & 36 & $1.40 \mathrm{E}-07$ & 2.10E-05 \\
\hline cell division & 20 & 7.90E-07 & 1.10E-04 \\
\hline spindle & 13 & $9.60 \mathrm{E}-06$ & 2.60E-03 \\
\hline cell cycle & 22 & 1.70E-05 & 7.80E-04 \\
\hline regulation of mitotic cell cycle & 11 & $2.90 \mathrm{E}-04$ & $1.50 \mathrm{E}-02$ \\
\hline microtubule cytoskeleton & 20 & $3.30 \mathrm{E}-03$ & 7.20E-02 \\
\hline cytoskeleton & 18 & 2.60E-02 & $3.80 \mathrm{E}-01$ \\
\hline cytoskeletal part & 23 & $1.00 \mathrm{E}-01$ & $6.00 \mathrm{E}-01$ \\
\hline cytoskeleton & 30 & 1.60E-01 & 7.50E-01 \\
\hline intracellular non-membrane-bounded organelle & 46 & 5.30E-01 & $9.70 \mathrm{E}-01$ \\
\hline non-membrane-bounded organelle & 46 & 5.30E-01 & $9.70 \mathrm{E}-01$ \\
\hline
\end{tabular}

known to be downregulated in higher-grade glial tumors such as miR-124 and miR-128. ${ }^{13}$

\section{Discussion}

Diagnostic uncertainty in discriminating between LGGs of oligocytic and astrocytic lineage remains a major challenge in neurooncology. Here, we analyzed TCGA data set in search of molecular biomarkers that could facilitate discrimination. The key findings of our study are as follows: 1) oligodendrogliomas are more likely to exhibit the G-CIMP phenotype relative to low-grade astrocytomas; 2) relative to oligodendrogliomas, low-grade astrocytomas exhibit a higher expression of genes related to mitosis, replication, and inflammation; and 3) low-grade astrocytic tumors harbor miRNA profiles similar to those previously described for glioblastoma tumors. Notably, such miRNA profiles were not observed in oligodendrogliomas. These molecular features may augment conventional histopathology in discriminating oligodendrogliomas from low-grade astrocytomas.
The G-CIMP phenotype was initially described by Noushmehr et al. ${ }^{20}$ as a pattern of epigenetic modification that is found in a subset of glioblastomas. These tumors exhibit increased methylation of cytosine in $\mathrm{CpG}$ islands that are often found in the promoter regions of transcribed genes. ${ }^{3}$ It is thought that promoter methylation silences critical genes that mediate glioblastoma aggressiveness and therapeutic resistance. Consequently, G-CIMP-positive glioblastomas have a better prognosis than glioblastomas without the G-CIMP phenotype. ${ }^{20}$ Our observation that oligodendrogliomas are more likely to harbor the G-CIMP phenotype is largely consistent with the more favorable prognosis of this LGG relative to low-grade astrocytic tumors. Five-year survival for patients with astrocytic LGGs is $56 \%$ compared with $74 \%$ in those with predominantly oligodendritic gliomas. ${ }^{33}$ Some oligodendrogliomas with $1 \mathrm{p} / 19 \mathrm{q}$ deletions are still slower growing tumors with even more indolent courses. ${ }^{25}$

The increased expression of mRNAs related to mitosis and inflammation in low-grade astrocytomas relative to ol- 


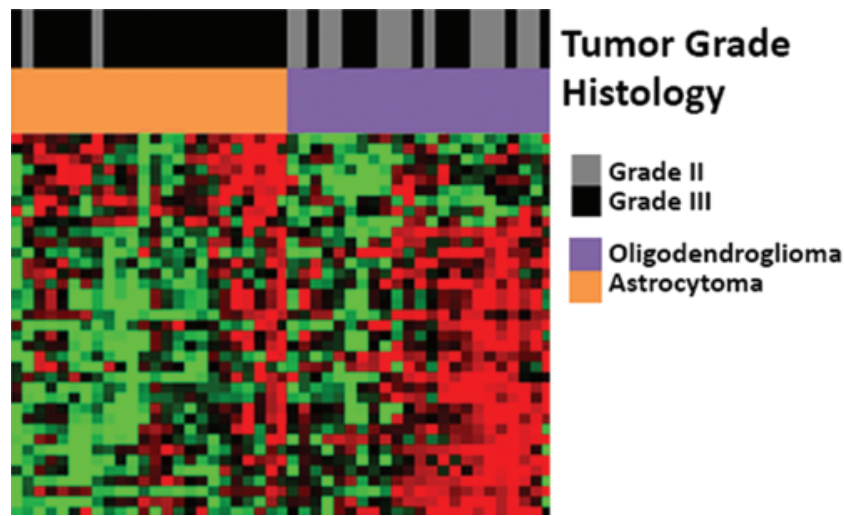

Fig. 4. Differential expression of miRNA from astrocytomas and oligodendrogliomas. Direct comparison between the 2 tumor types revealed 37 miRNAs with a minimum mean fold change of 2 . Tumor types (oligodendroglioma vs astrocytoma) are distinguished with a color code at the top of the panel: orange, astrocytoma; and purple, oligodendroglioma. Heatmap demonstrating the miRNA gene expression normalized for each gene using a color scale, with red representing higher levels of expression and green indicating lower levels.

igodendrogliomas is also consistent with the more aggressive clinical course of the astrocytomas. ${ }^{29,33}$ The increased expression of mRNAs related to mitosis probably mirrors increased rates of tumor proliferation. ${ }^{8}$ Increased expression of inflammatory cytokines in glioblastoma specimens has been associated with worsened overall survival. ${ }^{31,34,36}$ Emerging evidence suggests that the presence of inflammatory cytokines may enhance glioblastoma growth as well as suppress apoptotic and senescent processes within glioblastomas. ${ }^{6,36}$ The inflammatory markers upregulated in astrocytomas in the current analysis may reflect the increased number of microglia in astrocytic gliomas relative to oligodendrogliomas ${ }^{7,26}$ or the inherent expression of cytokines by astrocytic gliomas. ${ }^{12,19,35,36}$

Aberrant miRNA expression is a universal theme during carcinogenesis. ${ }^{16}$ Moreover, the pattern of miRNA expression often marks the lineage of origin with regard to the tumor. ${ }^{15,17}$ In this context, it is gratifying that we uncovered distinct miRNA profiles in astrocytic and oligodendrocytic LGGs. Several of the miRNAs we found to be expressed at higher levels in the astrocytomas as compared with the oligodendrogliomas have been previously described as key contributors to glioblastoma formation, including miR21.2,22 Conversely, many of the miRNAs downregulated in astrocytomas relative to oligodendrogliomas have been shown to be required in maintaining astrocytic differentiation, including miR-124 ${ }^{4}$ and $\mathrm{miR}-128 .^{21}$

\section{Conclusions}

Oligodendrogliomas and low-grade astrocytomas exhibit distinctly different molecular characteristics including G-CIMP status, mRNA expression, and miRNA profiles. Incorporation of these molecular platforms with existing molecular markers, such as IDH1 mutation, TP53 mutation, and $1 \mathrm{p} 19 \mathrm{q}$ status, should facilitate accurate and reliable pathological diagnosis of LGGs.

\section{Disclosure}

The authors report no conflict of interest concerning the mate-
TABLE 2: Differentially expressed miRNAs in astrocytomas relative to oligodendrogliomas*

\begin{tabular}{lc}
\hline \multicolumn{1}{c}{ miRNA } & Fold Change \\
\hline hsa-miR-455 & 2.501695 \\
hsa-miR-204 & 2.474307 \\
hsa-miR-200a & 2.417406 \\
hsa-miR-21 & 2.313565 \\
hsa-miR-937 & 2.312845 \\
hsa-miR-1262 & 2.197591 \\
hsa-miR-887 & 2.055209 \\
hsa-miR-577 & 2.025004 \\
hsa-miR-1251 & 2.015545 \\
hsa-miR-128-2 & 0.489822 \\
hsa-miR-451 & 0.482832 \\
hsa-miR-126 & 0.479343 \\
hsa-miR-1247 & 0.475773 \\
hsa-miR-486 & 0.475747 \\
hsa-miR-378 & 0.467384 \\
hsa-miR-124-1 & 0.454637 \\
hsa-miR-124-2 & 0.450175 \\
hsa-miR-1274b & 0.446444 \\
hsa-miR-592 & 0.444133 \\
hsa-miR-124-3 & 0.441731 \\
hsa-miR-129-2 & 0.440461 \\
hsa-miR-145 & 0.439702 \\
hsa-miR-551b & 0.433424 \\
hsa-miR-320b-2 & 0.430294 \\
hsa-miR-153-1 & 0.429561 \\
hsa-miR-153-2 & 0.427691 \\
hsa-miR-1224 & 0.411144 \\
hsa-miR-129-1 & 0.400323 \\
hsa-miR-139 & 0.390977 \\
hsa-miR-133a-1 & 0.379083 \\
hsa-miR-105-2 & 0.363826 \\
hsa-miR-203 & 0.357636 \\
\hline hsa-miR-1-2 & 0.349742 \\
\hline hsa-105-1 & 0.321588 \\
\hline
\end{tabular}

${ }^{*}$ hsa $=$ Homo sapiens.

rials or methods used in this study or the findings specified in this paper.

Author contributions to the study and manuscript preparation include the following. Conception and design: Carter, Chen. Acquisition of data: Muller. Analysis and interpretation of data: Gonda, Goyal, Chen. Drafting the article: Gonda, Cheung, Muller, Goyal. Critically revising the article: Gonda, Chen. Reviewed submitted version of manuscript: Goyal. Approved the final version of the manuscript on behalf of all authors: Gonda. Statistical analysis: Gonda. Study supervision: Carter, Chen. 


\section{D. Gonda et al.}

\section{References}

1. Cancer Genome Atlas Research Network: Comprehensive genomic characterization defines human glioblastoma genes and core pathways. Nature 455:1061-1068, 2008

2. Chan JA, Krichevsky AM, Kosik KS: MicroRNA-21 is an antiapoptotic factor in human glioblastoma cells. Cancer Res 65: 6029-6033, 2005

3. Deaton AM, Bird A: CpG islands and the regulation of transcription. Genes Dev 25:1010-1022, 2011

4. Fowler A, Thomson D, Giles K, Maleki S, Mreich E, Wheeler $\mathrm{H}$, et al: miR-124a is frequently down-regulated in glioblastoma and is involved in migration and invasion. Eur $\mathbf{J}$ Cancer 47:953-963, 2011

5. Fu X, Han Y, Wu Y, Zhu X, Lu X, Mao F, et al: Prognostic role of microRNA-21 in various carcinomas: a systematic review and meta-analysis. Eur J Clin Invest 41:1245-1253, 2011

6. Goswami S, Gupta A, Sharma SK: Interleukin-6-mediated autocrine growth promotion in human glioblastoma multiforme cell line U87MG. J Neurochem 71:1837-1845, 1998

7. Graeber MB, Scheithauer BW, Kreutzberg GW: Microglia in brain tumors. Glia 40:252-259, 2002

8. Gupta M, Djalilvand A, Brat DJ: Clarifying the diffuse gliomas: an update on the morphologic features and markers that discriminate oligodendroglioma from astrocytoma. Am J Clin Pathol 124:755-768, 2005

9. Huang W, Sherman BT, Lempicki RA: Bioinformatics enrichment tools: paths toward the comprehensive functional analysis of large gene lists. Nucleic Acids Res 37:1-13, 2009

10. Huang W, Sherman BT, Lempicki RA: Systematic and integrative analysis of large gene lists using DAVID bioinformatics resources. Nat Protoc 4:44-57, 2009

11. Ichimura K, Pearson DM, Kocialkowski S, Bäcklund LM, Chan R, Jones DT, et al: IDH1 mutations are present in the majority of common adult gliomas but rare in primary glioblastomas. Neuro Ooncol 11:341-347, 2009

12. Jehs T, Faber C, Juel HB, Nissen MH: Astrocytoma cells upregulate expression of pro-inflammatory cytokines after coculture with activated peripheral blood mononuclear cells. APMIS 119:551-561, 2011

13. Karsy M, Arslan E, Moy F: Current progress on understanding MicroRNAs in glioblastoma multiforme. Genes Cancer 3: $3-15,2012$

14. Kim L, Hochberg FH, Thornton AF, Harsh GR IV, Patel H, Finkelstein D, et al: Procarbazine, lomustine, and vincristine (PCV) chemotherapy for grade III and grade IV oligoastrocytomas. J Neurosurg 85:602-607, 1996

15. Kim TM, Huang W, Park R, Park PJ, Johnson MD: A developmental taxonomy of glioblastoma defined and maintained by MicroRNAs. Cancer Res 71:3387-3399, 2011

16. Kong YW, Ferland-McCollough D, Jackson TJ, Bushell M: microRNAs in cancer management. Lancet Oncol 13:e249e258, 2012

17. Lu J, Getz G, Miska EA, Alvarez-Saavedra E, Lamb J, Peck D, et al: MicroRNA expression profiles classify human cancers. Nature 435:834-838, 2005

18. Matthews S, Succar P, Jelinek H, McParland B, Buckland M, McLachlan CS: Diagnosis of oligodendroglioma: molecular and classical histological assessment in the twenty-first century. Asia Pac J Clin Oncol 8:213-216, 2012

19. Nitta T, Ebato M, Sato K, Okumura K: Expression of tumour necrosis factor-alpha, -beta and interferon-gamma genes within human neuroglial tumour cells and brain specimens. Cytokine 6:171-180, 1994

20. Noushmehr H, Weisenberger DJ, Diefes K, Phillips HS, Pujara K, Berman BP, et al: Identification of a $\mathrm{CpG}$ island meth- ylator phenotype that defines a distinct subgroup of glioma. Cancer Cell 17:510-522, 2010

21. Papagiannakopoulos T, Friedmann-Morvinski D, Neveu P, Dugas JC, Gill RM, Huillard E, et al: Pro-neural miR-128 is a glioma tumor suppressor that targets mitogenic kinases. Oncogene 31:1884-1895, 2012

22. Papagiannakopoulos T, Shapiro A, Kosik KS: MicroRNA-21 targets a network of key tumor-suppressive pathways in glioblastoma cells. Cancer Res 68:8164-8172, 2008

23. Perry A: Oligodendroglial neoplasms: current concepts, misconceptions, and folklore. Adv Anat Pathol 8:183-199, 2001

24. Reifenberger G, Louis DN: Oligodendroglioma: toward molecular definitions in diagnostic neuro-oncology. J Neuropathol Exp Neurol 62:111-126, 2003

25. Rodriguez FJ, Giannini C: Oligodendroglial tumors: diagnostic and molecular pathology. Semin Diagn Pathol 27:136145,2010

26. Roggendorf W, Strupp S, Paulus W: Distribution and characterization of microglia/macrophages in human brain tumors. Acta Neuropathol 92:288-293, 1996

27. Saeed AI, Sharov V, White J, Li J, Liang W, Bhagabati N, et al: TM4: a free, open-source system for microarray data management and analysis. Biotechniques 34:374-378, 2003

28. Schiffer D, Dutto A, Cavalla P, Bosone I, Chio A, Villani R, et al: Prognostic factors in oligodendroglioma. Can J Neurol Sci 24:313-319, 1997

29. Shaw EG, Scheithauer BW, O'Fallon JR: Supratentorial gliomas: a comparative study by grade and histologic type. J Neurooncol 31:273-278, 1997

30. Smith JS, Perry A, Borell TJ, Lee HK, O'Fallon J, Hosek SM, et al: Alterations of chromosome arms $1 p$ and $19 q$ as predictors of survival in oligodendrogliomas, astrocytomas, and mixed oligoastrocytomas. J Clin Oncol 18:636-645, 2000

31. Tchirkov A, Khalil T, Chautard E, Mokhtari K, Véronèse L, Irthum B, et al: Interleukin- 6 gene amplification and shortened survival in glioblastoma patients. Br J Cancer 96:474-476, 2007

32. Verhaak RG, Hoadley KA, Purdom E, Wang V, Qi Y, Wilkerson MD, et al: Integrated genomic analysis identifies clinically relevant subtypes of glioblastoma characterized by abnormalities in PDGFRA, IDH1, EGFR, and NF1. Cancer Cell 17:98110,2010

33. Walker C, Haylock B, Husband D, Joyce KA, Fildes D, Jenkinson $\mathrm{MD}$, et al: Clinical use of genotype to predict chemosensitivity in oligodendroglial tumors. Neurology 66:1661-1667, 2006

34. Xie Q, Thompson R, Hardy K, DeCamp L, Berghuis B, Sigler $\mathrm{R}$, et al: A highly invasive human glioblastoma pre-clinical model for testing therapeutics. J Transl Med 6:77, 2008

35. Yamanaka R, Tanaka R, Saitoh T, Okoshi S: Cytokine gene expression on glioma cell lines and specimens. J Neurooncol 21:243-247, 1994

36. Zhu VF, Yang J, Lebrun DG, Li M: Understanding the role of cytokines in Glioblastoma Multiforme pathogenesis. Cancer Lett 316:139-150, 2012

Manuscript submitted October 23, 2012.

Accepted December 11, 2012.

Please include this information when citing this paper: DOI: 10.3171/2012.12.FOCUS12351.

Address correspondence to: Clark C. Chen, M.D., Ph.D., Division of Neurosurgery, University of California, San Diego, 3855 Health Science Drive \#0987, La Jolla, California 92093-0987. email: clark chen@ucsd.edu. 